\title{
IHV: A NEW LONG-TERM GEOMAGNETIC INDEX
}

\author{
Leif Svalgaard ${ }^{1}$, Edward W. Cliver ${ }^{2}$, and Philippe Le Sager ${ }^{3}$ \\ ${ }^{I}$ Easy Toolkit, Inc., 6927 Lawler Ridge, Houston TX 77055-7010, USA \\ ${ }^{2}$ Space Vehicles Directorate, Air Force Research Laboratory, Hanscom AFB, MA 01731, USA \\ ${ }^{3}$ Prairie View A\&M University, Solar Observatory, PO Box 307, Prairie View, TX 77446, USA
}

\begin{abstract}
We derive a new daily index of geomagnetic activity, the Inter-Hour Variability index (IHV), for investigations of the long-term variability of the solar wind-magnetosphere system. The IHV index is used to successfully reconstruct yearly-averages of the range indices am, ap, and $a a$ from 1959 through 2000. When we attempt to reconstruct the $a a$ index back to 1901, however, the reconstructed $a a$ lies above the observed $a a$ for years before 1957 , with the difference between the two curves being the greatest $(\sim 5-10 \mathrm{nT})$ during the first two decades of the $20^{\text {th }}$ century.
\end{abstract}

\section{Introduction}

The long time series of the $a a$ index (Mayaud, 1972) is commonly used to investigate geomagnetic activity and the Sun-Earth connection. The validity of any analysis based on $a a$ rests on the assumed constancy of the calibration of this index over time. Svalgaard et al. (2003) have presented evidence of the non-constancy of the $a a$ calibration. The present paper seeks to explain and elaborate on the methodology of that study.

It was realized long ago that solar electromagnetic radiation (primarily EUV) and solar "corpuscular" radiation (what we today call the "solar wind") give rise to different classes of fluctuations of the geomagnetic field. EUV radiation creates and maintains the ionospheric layer and the tidal winds in the atmosphere. Their combined effect produces the regular daily $\mathrm{Sq}$ variation by dynamo action, which is well understood and has been successfully modeled (Wagner, 1980; Richmond, 1995). On the other hand, the less well-understood solar wind induced activity is measured by geomagnetic indices such as $a a$. Derivation of a long-term index like $a a$ involves both the ability of the observer to correctly identify the fluctuations caused by the solar wind and the availability of appropriate conversion tables. As stations or instruments change over time, new conversion tables have to be drawn up and intercalibrated with the previous tables. The entire process cannot easily be duplicated and the quality of the index values is difficult to gauge. The long time-series of hourly values of the magnetic components from several stations (potentially going back to about 1835) form a rationale for investigating if an index can be constructed from them with the following properties:

- Easy to understand (existing indices are derived via a process that is convoluted, intricate, and in its details largely unknown - to most researchers)

- Completely objective (no subjective determination of baselines, quiet background, or the like)

- Easy to duplicate by other researchers for verification purposes

- Physically quantitative (expressed directly in or proportional to magnetic variations measured in nT)

- Suitable for studies of long-term variations (on time scales of weeks and longer) 
- Having the property that its limitations are clearly stated and understood

- Constant calibration over time for any given station (not requiring conversion tables or daily or seasonal adjustment tables)

\section{The IHV index}

The fluctuations sensitive to solar EUV radiation are mainly confined to local day hours and are effectively absent deep into the night. This suggests that we can derive a new index of solar wind type activity that is suitable for analysis of long-term behaviour by only using data from 6 hours centred on local midnight. Figure 1 shows the average daily variation of the horizontal component at the mid-latitude station Cheltenham/Fredericksburg.

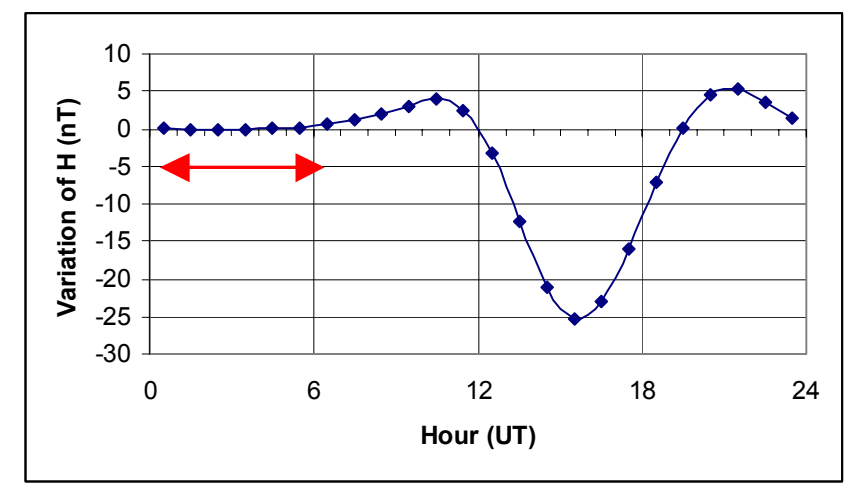

Fig. 1. The average daily variation of the H-component at Cheltenham / Fredericksburg for the years 1901-2000. The definition of the local night-time is shown by the horizontal arrow. The $S q$ variation begins to be felt from $6^{\mathrm{h}}$ UT, has maximum effect close to local noon, and has effectively ceased at $24^{\mathrm{h}}$ UT. Zero-level has been set at $3: 30^{\mathrm{h}}$ UT.

We calculate for each station the sum of the differences (taken without regard to their sign) between each hourly value and the next of the horizontal component of the geomagnetic field for the six hours around local midnight, then divide the sum by 6 to get a measure (the $I H V$ index) of the average inter-hour variability for each day. Formally:

$$
I H V^{\mathrm{H}}(\mathrm{day})=\sum_{h=h 1}^{h=h 2}\left[\operatorname{abs}\left(H_{h}-H_{h+1}\right)\right] /(h 2-h 1+1)
$$

Where the hourly index $h$ runs from $h 1$ through $h 2$ (if the hourly mean refers to the hour from 0:00 ${ }^{\mathrm{h}}$ UT to $1: 00^{\mathrm{h}} \mathrm{UT}$, we assign to it the hourly index 0 , thus $h l=0$ and $h 2=5$ for Cheltenham/Fredericksburg). If $h 1$ and $h 2$ are on opposite sides of $0^{\mathrm{h}} \mathrm{UT}$, the hourly index is taken modulo 24 . We could define similar indices for the D, Z, X, or $\mathrm{Y}$ components. If we omit the superscript (the ${ }^{\mathrm{H}}$ in $I H V^{\mathrm{H}}$ ), the $\mathrm{H}$-component is assumed. This measure satisfies the criteria set out above.

The requirement of "constant calibration" can be relaxed when simultaneous observations exist for several geomagnetic stations or observatories allowing a simple "cross calibration" provided it is clear that the index derived from one station differs but by a constant factor from that derived from another station. Cheltenham and Fredericksburg are located very close to each other (only a few miles apart) and operated simultaneously for 273 days during 1956. The $I H V$ index calculated at both stations for these 273 days have a correlation coefficient of 0.994. The very strong correlation justifies calibrating the $I H V$-index values derived from (the older station) Cheltenham to corresponding values as were they derived from Fredericksburg by multiplying them by 0.970 . Short data gaps can be filled in from the $I H V$ values calculated from other stations calibrated the same way. This allows us to construct an unbroken series of $I H V$ values from 1901 through 2000. 


\section{Reconstruction of $a m, a p$ and $a a$ indices}

The am geomagnetic activity index (Mayaud, 1980), derived for years beginning with 1959 from a reasonably dense network of midlatitude stations in both hemispheres, is generally considered the most "accurate" geomagnetic index. In this section, we investigate how our $I H V$-index correlates with the am-index. Figure 2 shows the relationship between yearly average values of the $I H V$-index for Fredericksburg and yearly average values of the $a m$-index. The correlation is highest $\left(\mathrm{R}^{2}=0.943\right)$ for the line with an offset, and:

$$
a m=5.6131 * I H V_{F R D}-4.4941
$$

Using Eq. (2), we can now reconstruct am. The result is shown in Figure 3.

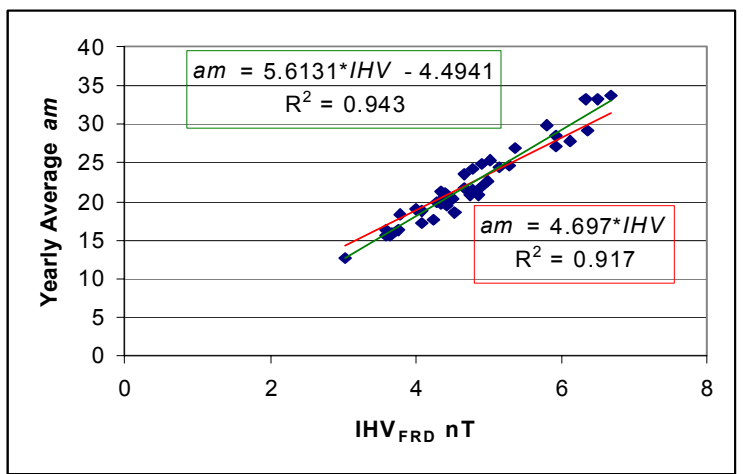

Fig. 2. Yearly average of the am-index for each year during 1959-2000 compared to the yearly average $I H V$-index for Fredericksburg. The values used for calculation of am refer to the same UT-intervals used in the calculation of $I H V$. Lines with and without an offset are least-squared fitted as shown.

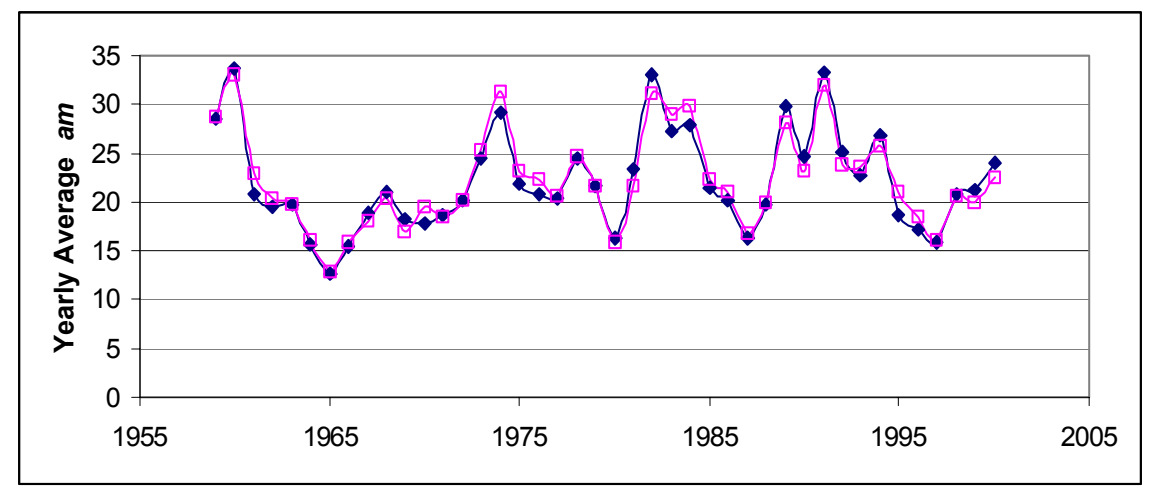

Fig. 3. Reconstructed yearly average am-index (pink squares) for the interval 1959-2000. The observed values of the am-index are shown as blue diamonds

Having successfully reconstructed the $a m$-index, we can apply the same method to reconstructing the $a p$-index for the same interval. Figure 4 shows the relationship between yearly average values of the $I H V$-index for Fredericksburg and yearly average values of the $a p$-index. The correlation $\left(\mathrm{R}^{2}=0.926\right)$ is almost as high as for $a m$, and:

$$
a p=4.5322 * I H V_{F R D}-5.9911
$$


Using Eq. (3) we can now reconstruct ap. The result is shown in Figure 5. No significant differences are apparent and no significant long-term trend either.

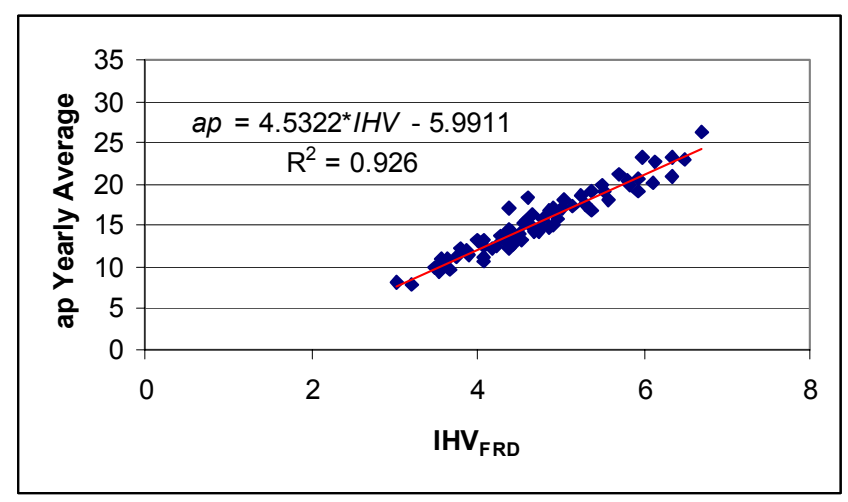

Fig. 4. Yearly average of the $a p$-index for each year during 1959-2000 compared to the yearly average $I H V$-index for Fredericksburg. The values used for calculation of ap refer to the same UT-intervals used in the calculation of $I H V$. The leastsquared fit best line is shown.

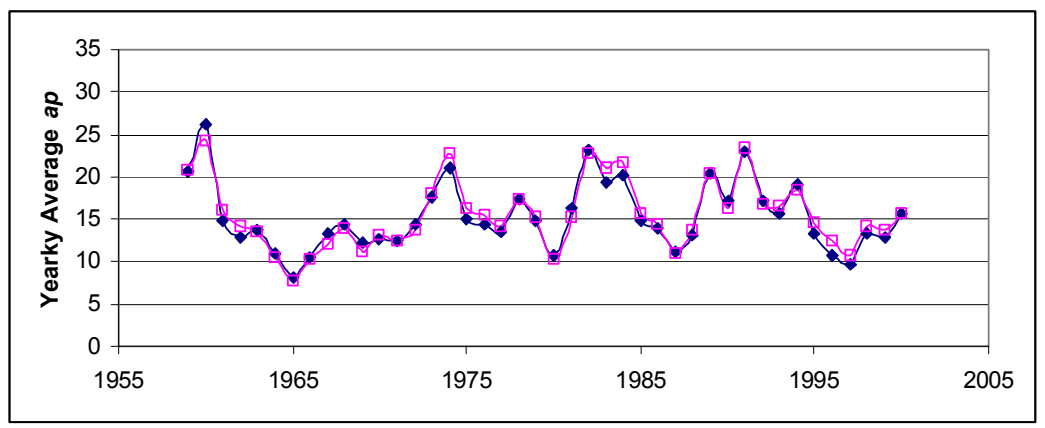

Fig. 5. Reconstructed ap-index (pink squares) for the interval 1959-2000 compared to the observed values (blue diamonds). Yearly averages are shown.

We can apply the same method to reconstruct the $a a$-index for the same interval. Figure 6 shows the relationship between yearly average values of the $I H V$-index for Fredericksburg and yearly average values of the $a a$-index. The correlation $\left(\mathrm{R}^{2}=0.923\right)$ is almost as high as for $a m$, and:

$$
a a=4.8304 * I H V_{F R D}-1.7267
$$

Using Eq. (4), we can now reconstruct $a a$. The result is shown in Figure 7. There are no significant differences and no significant long-term trend.

Judging from Figures 3, 5, and 7 we can with fair success reconstruct all the range indices $a m$, $a p$, and $a a$ for the interval 1959-2000, at least their yearly average values. We deliberately chose the same interval for all three indices in order to be able to compare the efficacy of the reconstructions. So far, no significant differences are apparent. It does indeed seem that the $I H V$-index can be used as a basis for reconstruction of the standard geomagnetic indices. The very direct and straightforward method of determining the $I H V$ values and thereby reconstructed standard indices seems to carry promise of extending the reconstructions as far back in time as we have hourly values available for mid-latitude stations, some 150 years or more.

Bolstered by our success in reconstructing all three standard mid-latitude indices for the last forty years, we employ the exact same method and reconstruct $a a$ for the past one hundred years. The surprising result is shown in Figure 8. 


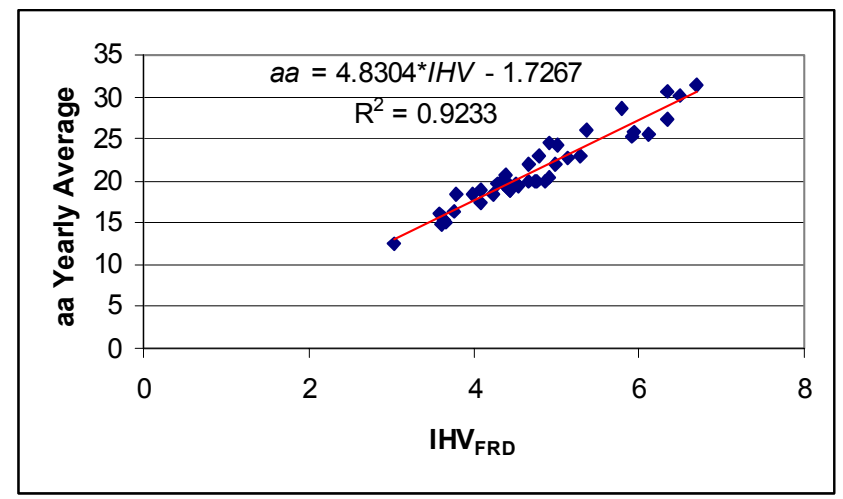

Fig. 6. Yearly average of the aa-index for each year during 1959-2000 compared to the yearly average $I H V$-index for Fredericksburg. The values used for calculation of aa refer to the same UT-intervals used in the calculation of $I H V$. The least-squared fit best line is shown.

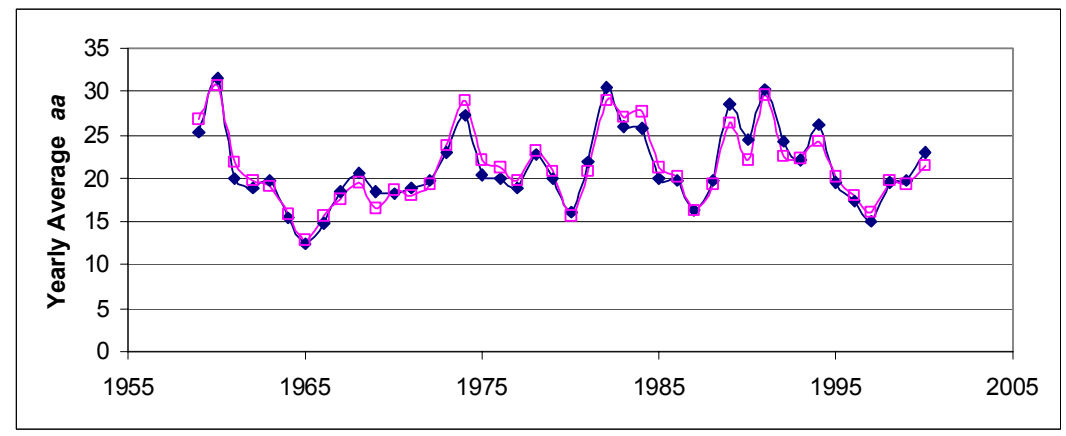

Fig. 7. Reconstructed aa-index (pink squares) for the interval 1959-2000 compared to the observed values (blue diamonds).

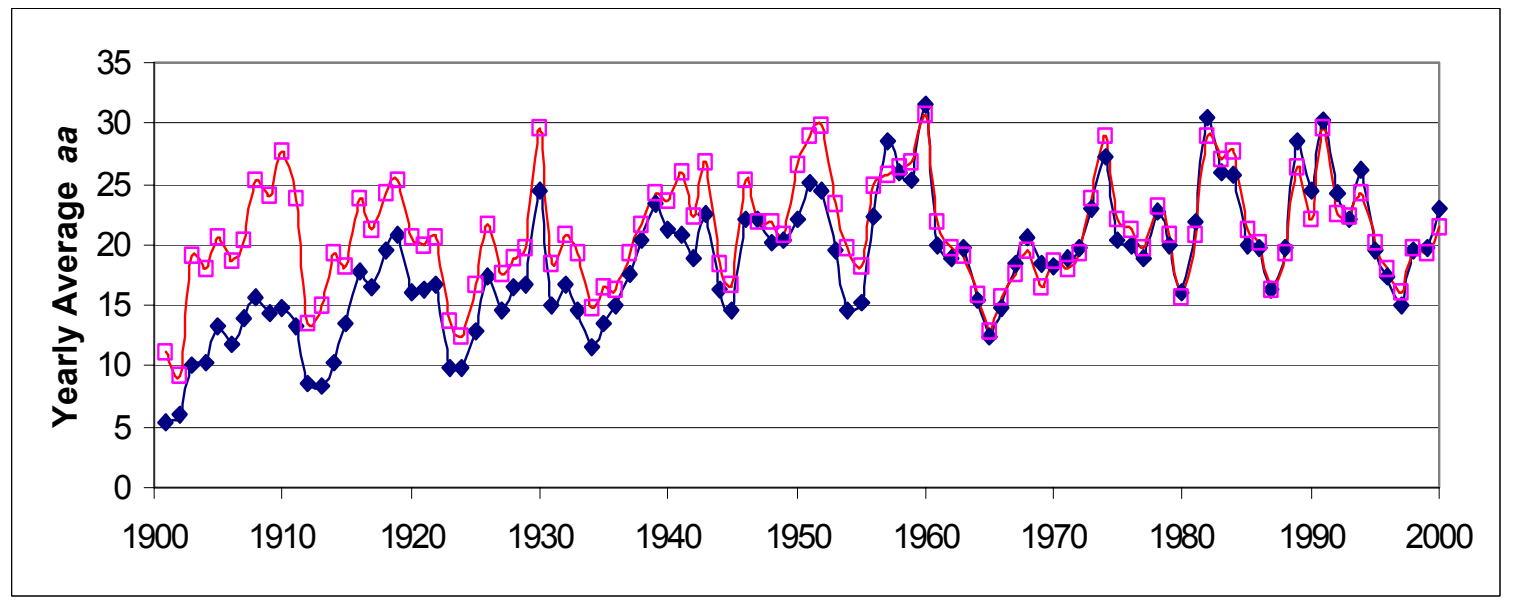

Fig. 8. Observed (blue diamonds) and reconstructed (pink squares) yearly average values of the $a a$-index (during the two three-hour periods from 0 to $6 \mathrm{UT}$ ). The Reconstruction was based on $I H V$ values from Cheltenham and Fredericksburg (CHL reduced to FRD levels) and on Eq. (4). 
For the last forty years, the observed and the reconstructed values of $a a$ track each other rather closely. Before 1957, however, the two values diverge progressively as we go back in time to the point of differing by almost a factor of two in the early part of the $20^{\text {th }}$ century. Some of the discrepancy between the two curves can be related to equipment/station changes. Clilverd et al. (2002) note that the magnetograph installed at Abinger in 1938 has a sensitivity about half that of the old instrument. This makes low $\mathrm{K}$ values more difficult to identify, consistent with the higher $a a$ values observed after that year. The year 1957, before which the two curves diverge, corresponds to the move of the English station from Abinger to Hartland. While these factors contribute to the differences between observed and reconstructed $a a$, more work will be required both to substantiate these differences and to determine their causes.

\section{Conclusion}

We have derived a new simplified daily index of geomagnetic activity that is well suited for studies of the long-term variability of solar-terrestrial interactions. The key feature of the inter-hour variability (IHV) index is its use of local night time hours (at the cost of high time resolution) to remove the EUV-induced regular variation.

When we use the $I H V$ index to reconstruct the $a a$ index - the standard index used for long-term studies of geomagnetic variability - for the last 100 years, we find that the reconstructed $a a$ lies above the observed $a a$ before 1957 , with the greatest discrepancy $(\sim 5-10 \mathrm{nT})$ occurring for the first two decades of the $20^{\text {th }}$ century. Thus any claim based on the rise of $a a$ (as observed and reported by ISGI (Service International des Indices Geomagnetique)) since 1900 may be inaccurate. Implications of the non-constant calibration of the $a a$ index were addressed in Svalgaard et al. (2003).

\section{REFERENCES}

Clilverd, M. A., T. D. G. Clark, E. Clarke, H. Rishbeth, and T. Ulich, The causes of long-term change in the aa index, J. Geophys. Res., 107(A12), 1441, doi:10.1029/2001JA000501, 2002.

Mayaud, P.N., The $a a$ indices: a 100-year series characterising the magnetic activity, J. Geophys. Res., 72, 68706874, 1972.

Mayaud, P.N., Derivation, Meaning, and Use of Geomagnetic Indices, American Geophysical Union, Washington D.C., 1980.

Svalgaard, L., E.W. Cliver, and P. Le Sager, No doubling of the Sun's coronal magnetic field during the last 100 years, Nature (submitted), 2003.

Richmond, A. D., The ionospheric wind dynamo: effects of its coupling with different atmospheric regions, in The upper mesosphere and lower thermosphere: a review of experiment and theory, Geophysical monograph 87, AGU, 49-65, 1995.

Wagner, C.-U., D. Möhlmann, K. Schafer, V. M. Mishin, and M. I. Matveev, Large scale electric fields and currents and related geomagnetic variations in the quiet plasmasphere, Space Sci. Rev., 26, 391-446, 1980.

E-mail address of L. Svalgaard leif@leif.org

E-mail address of E. Cliver Edward.Cliver@hanscom.af.mil

E-mail address of P. Le Sager philippe lesager@pvamu.edu 